\title{
ACCEPTOR IN QUANTUM DOT IN CUBIC SEMICONDUCTORS
}

\author{
P. JANISZEWSKI AND M. SUFFCZYŃSKI
}

Institute of Physics, Polish Academy of Sciences Al. Lotników 32/46, 02-668 Warszawa, Poland

(Received July 18, 1995)

\begin{abstract}
Energy levels and oscillator strengths for transitions between the lowest states of an acceptor in a quantum dot of finite potential barrier in cubic semiconductors have been computed in the effective-mass approximation. The degeneracy of the valence band in cubic semiconductors was taken into account in the spherical approximation. Variational envelope functions consisted of a finite basis of exponentials, and had to satisfy appropriate boundary conditions to ensure the hermiticity of the Ifamiltonian matrix. In typical cubic semiconductors we have found enhanced values, by an order of magnitude, of oscillator strengths for the acceptor optical transitions in the dots of radii comparable to the acceptor diameter.
\end{abstract}

PACS numbers: 73.20.Dx, 73.61.Ey, 78.66.Fd

\section{Introduction}

Studies of superlattices of zero-dimensional quantum dots require a systematic quantum mechanical calculations since these low-dimensional structures are now being constructed under a reliable supervision of their parameters. When the dot radius is comparable to the carrier wave function extension, the properties of carriers, bound to an impurity, in a quantum dot can be considerably different from those in the bulk. An impurity in a quantum-well, -wire or -dot has an energy spectrum determined not only by the Coulomb potential of the impurity center but also by the confining potential of the barrier, and by the structure of subbands of two-, one- or zero-dimensional single particle states, respectively [1-4].

\section{The effective-mass equations}

We study an impurity located at the center of a spherical quantum dot of radius $R$ in a cubic semiconductor " $I$ " embedded in a semiconductor " $E$ " with a potential barrier of finite height. In the effective-mass approximation [1-6], for a shallow acceptor impurity the mixing of the light and heavy hole states in the 
degenerate valence band necessitates the use of the matrix IIamiltonian. In cubic semiconductors $I, E$ the Luttinger effective-mass parameters of the highest valence band edge, with $J=3 / 2$, have the respective values $\gamma_{1}^{I, E}, \gamma_{2}^{I, E}, \gamma_{3}^{I, E}$. The static dielectric constant $\varepsilon_{0}$ is assumed to be the same in the dot as in the bulk outside of the dot, thus any dielectric mismatch [6] is neglected. With an effective mass $m^{*}$ the effective Bohr radius $a_{0}^{*}=\hbar^{2} \varepsilon_{0} / e^{2} m^{*}$ and the effective Rydberg $R y d^{*}=e^{4} m^{*} / 2 \hbar^{2} \varepsilon_{0}^{2}$ are units of length and energy, respectively. We use the Baldereschi and Lipari [5] spherical-model IIamiltonian in the limit of large spin-orbit splitting between the valence bands. With $\bar{\gamma}^{I, E}=\left(2 \gamma_{2}^{I, E}+3 \gamma_{3}^{I, E}\right) / 5$ we restrict the calculations to the spherical approximation based on simplifying assumption of the isotropy of the hole energy band: $\gamma_{2}^{I, E}=\gamma_{3}^{I, E}=\bar{\gamma}^{I, E}$. On these assumptions, the effective-mass kinetic energy Hamiltonian can be expressed by scalar product of the irreducible tensors [2-5, 7-9] of momentum $p$ and angular momentum $J$.

A singly charged acceptor, $Z=1$, situated at the center of a dot of radius $R$ has the potential energy

$$
V^{I, E}(r)=V_{\mathrm{C}}-V_{\mathrm{B}}^{I, E}=-Z e^{2}\left(\varepsilon_{0} r\right)^{-1}-V_{\mathrm{B}}^{I, E},
$$

which is the sum of the acceptor Coulomb potential and of the potential of the dot barrier. We assume $V_{\mathrm{B}}^{E}=0$ for $r>R$. Therefore the IIamiltonian can be written $[2-6,8,9]$

$$
H^{I, E}=\left(\gamma_{1}^{I, E} / 2 m_{0}\right)\left[p^{2}-\mu^{I, E}\left(P^{(2)} \cdot J^{(2)}\right)\right]+V_{\mathrm{C}}-V_{\mathrm{B}}^{I, E} .
$$

The $P^{(2)}$ and $J^{(2)}$ are the second-order irreducible tensor operators [2-5, 7-9] of the momentum and $J=3 / 2$ spin angular momentum, respectively. The term proportional to the parameter $\mu^{I, E}=2 \bar{\gamma}^{I, E} / \gamma_{1}^{I, E}$, corresponds to the spin-orbit coupling term. A shallow bound state of the hole has spin $J=3 / 2$ and an orbital angular momentum $\boldsymbol{L}$. The total angular momentum is $\boldsymbol{F}=\boldsymbol{L}+\boldsymbol{J}$. The spin-orbit term couples the states with angular momentum quantum numbers $L_{2}$ and $L_{2}$ for which $\left|L_{1}-L_{2}\right|=0,2$. Therefore the hole envelope functions can be written in the form [2-6]

$$
\Phi\left(L, 3 / 2, F, F_{z}\right)=f_{1 j}(r)\left|L, 3 / 2, F, F_{z}\right\rangle+f_{2 j}(r)\left|L+2,3 / 2, F, F_{z}\right\rangle,
$$

where the kets $\left|L, J, F, F_{z}\right\rangle$ are eigenfunctions of the total angular momentum $\boldsymbol{F}$ in the $L-J$ coupling scheme and the four figures in the ket represent the eigenvalues of the operators $L^{2}, J^{2}, F^{2}$, and $F_{z}$, respectively. We use the simplified indices $(i, j)$ in place of the set of the angular momentum quantum numbers. For a given state we will also write the radial functions as a two-dimensional vector $\bar{f}=\left(f_{1 j}(r), f_{2 j}(r)\right)$. The radial functions $f_{1 j}(r)$ and $f_{2 j}(r)$ of the envelope have to satisfy the set of differential equations [2-5]

$$
\sum_{l=1,2}\left[H_{k l}-E_{j} \delta_{k l}\right] f_{l j}(r)=0, \quad[k ; l]=[1,2 ; 1,2],
$$

where

with

$$
H_{k l}=A_{k l} \mathrm{~d}^{2} / \mathrm{d} r^{2}+B_{k l} r^{-1} \mathrm{~d} / \mathrm{d} r+C_{k l} r^{-2}+V(r) \delta_{k l}
$$

$$
[A ; B ; C]_{11}=-\left(1+C_{1}\right) \gamma_{1}[1 ; 2 ;-L(L+1)],
$$




$$
\begin{aligned}
& {[A ; B ; C]_{22}=-\left(1-C_{1}\right) \gamma_{1}[1 ; 2 ;-(L+2)(L+3)],} \\
& {[A ; B ; C]_{12}=C_{2} \gamma_{1}[1 ; 2 L+5 ;(L+1)(L+3)],} \\
& {[A ; B ; C]_{21}=C_{2} \gamma_{1}[1 ;-(2 L+1) ; L(L+2)] .}
\end{aligned}
$$

In the effective length and energy units, with $m^{*}=m_{0}$,

$$
V(r)= \begin{cases}-2\left(r^{-1}+V_{\mathrm{B}}\right) & \text { for } r<R, \\ -2 r^{-1} & \text { for } r>R .\end{cases}
$$

The coefficients $C_{1}$ for the lowest acceptor states are listed in Table, and $C_{2}=\left[\mu^{2}-C_{1}^{2}\right]^{1 / 2}$.

\section{TABLE}

Coefficients in the hole radial Eqs. (2.5).

\begin{tabular}{c|c|c|c|c|c|c|c}
\hline \hline$L_{F}$ & $S_{3 / 2}$ & $P_{1 / 2}$ & $P_{3 / 2}$ & $P_{5 / 2}$ & $D_{1 / 2}$ & $D_{5 / 2}$ & $D_{7 / 2}$ \\
\hline$C_{1}$ & 0 & $\mu$ & $-4 \mu / 5$ & $\mu / 5$ & $\mu$ & $-5 \mu / 7$ & $2 \mu / 7$
\end{tabular}

\section{Boundary conditions}

We assume as boundary condition $[10,11]$ for the envelope functions $\Phi_{j}$ their continuity at the dot radius, $r=R$, allowing for the different values of the Luttinger effective-mass parameters inside and outside of the dot. To ensure the hermiticity of the effective-mass IIamiltonian matrix $[10,11]$ we compute the matrix elements of the matrix $D$ :

$$
\begin{aligned}
& -D_{11}=D_{22}=\frac{3}{2} C_{1} \gamma_{1}, \\
& D_{12}=(L+3) C_{2} \gamma_{1}, \\
& D_{21}=-L C_{2} \gamma_{1} .
\end{aligned}
$$

The matrices $A, B, C, D$, the coefficients $C_{1}, C_{2}$, the functions $\Phi_{j}$ and $\vec{f}$ in semiconductors $I, E$ depend on the respective values of the Luttinger parameters and have to bear the corresponding labels: $I$ or $E$. The hermiticity of the effective-mass Hamiltonian is ensured when, at the dot boundary, $r=R$, for each state $j$, the radial functions $f_{1 j}^{I, E}(r), f_{2 j}^{I, E}(r)$, written as a vector $\bar{f}^{I, E}$, satisfy $\bar{f}^{I}=\bar{f}^{E}$, and their radial derivatives satisfy at $r=R$ :

$$
\mathrm{d} \bar{f}^{I} / \mathrm{d} r=\left(A^{I}\right)^{-1}\left[A^{E} \mathrm{~d} \bar{f}^{E} / \mathrm{d} r+\left(D^{E}-D^{I}\right) \bar{f}^{E} / R\right] .
$$

\section{Variational functions}

To find a variational solution of Eqs. (2.4) we used an expansion of the $f_{1 j}(r)$ and $f_{2 j}(r)$ functions in a series of $N$ exponential functions $c_{i j n} \exp \left(-r q^{n} / R_{j \max }\right)$, with $i=1,2$ and $n=1,2, \ldots, N$. The number $N$ of basis exponential functions was between 5 and 20 for space inside of the dot, and between 3 and 10 outside of the dot. The value of the scale parameter $q$ was equal to 2 , in most cases. The values of the radius $R_{j \max }$ were found by trial and error to minimize the energy 
eigenvalue $E_{j}$. The two conditions for hermiticity of the IIamiltonian matrix, continuity of the envelope function and Eq. (3.2), allow to eliminate two from the $N$ components of each envelope, and thus to reduce the dimension of the secular determinant from $2 N$ to $2(N-2)$. It was advantageous to eliminate the components with coefficients $c_{i j n}$ of that part of the envelope function of Eq. (2.3) which corresponds to the region of the dot barrier potential in order to avoid appearance of large absolute values of matrix elements in the secular equation. For the component functions $f_{i j}(r)$ with $L>0$ one of the coefficients $c_{i j n}$ was adjusted so as to make $f_{i j}(0)=0$. We have computed the lowest and the first excited acceptor levels for the states enumerated in Table.

\section{Energy levels}

We calculated energy and envelope functions for the lowest levels of acceptor situated at the center of quantum dots in typical cubic semiconductors [12-17]. Computed lowest energy levels of an on-center acceptor, expressed in units of the rydberg $R y d_{\text {HH }}$ of the heavy hole, are presented in Fig. 1 as functions of the quantum-dot radius, expressed in units of the Bohr radius $a_{0 \mathrm{HH}}$ of the heavy hole with the effective mass $m^{*}=m_{\mathrm{HH}}=m_{0} /\left(\gamma_{1}-2 \gamma_{2}\right)$. Here the values $\gamma_{1}^{I}=6.4$, $\gamma_{2}^{I}=2.4, \gamma_{1}^{E}=4.8, \gamma_{2}^{E}=1.6$, are adopted, so that the heavy-hole mass is equal to $m_{\mathrm{HH}}^{I, E}=m_{0} / 1.6$. The energy differences between the successive levels increase with decreasing dot size, similarly as in the case of the two-dimensional quantum well $[8,9]$.

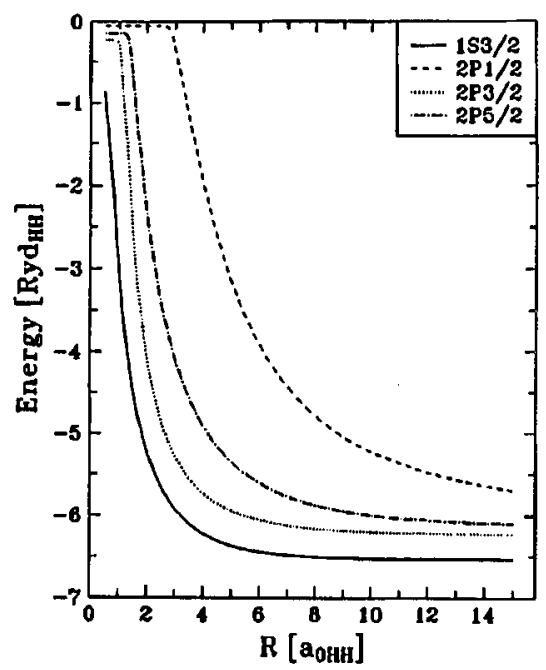

Fig. 1. The computed energy levels of an on-center acceptor, in units of the rydberg $R y d_{\mathrm{HH}}$ of the heavy hole, as function of the quantum-dot radius, expressed in units of the Bohr radius $a_{0 H \mathrm{H}}$ of the heavy hole, with $\gamma_{1}^{I, E}=1, \gamma_{2}^{I, E}=0.35$, and the potential barrier height $V_{\mathrm{B}}=6 \times R y d_{\mathrm{HH}}$. 


\section{Optical transitions}

For a calculation of the oscillator strengths $[8,9,18,19]$ for optical dipole transitions $f_{0 j}$ between the acceptor initial state $\Phi_{0}$ and an excited state $\Phi_{j}$, with the corresponding energy $E_{0}$ and $E_{j}$, respectively, the envelope functions have to be normalized. The normalization integral of the envelope functions, and the matrix element of the dipole moment, consist of the contribution from the space inside $(I)$ and outside $(E)$ of the dot

$$
\int \mathrm{d} \Omega\left\{\int_{0}^{R}\left|\Phi_{j}^{I}\right|^{2}+\int_{R}^{\infty}\left|\Phi_{j}^{E}\right|^{2}\right\} r^{2} \mathrm{~d} r=1 .
$$

The integration $\mathrm{d} \Omega$ is over the full solid angle. For the value of the Luttinger parameter $\gamma_{1}$ an average in the ground state was taken

$$
\gamma_{1}^{0}=\gamma_{1}^{I} c^{I}+\gamma_{1}^{E}\left(1-c^{I}\right)
$$

where

$$
c^{I}=\left\langle\Phi_{0}^{I} \mid \Phi_{0}^{I}\right\rangle=\int \mathrm{d} \Omega \int_{0}^{R}\left|\Phi_{0}^{I}\right|^{2} r^{2} \mathrm{~d} r .
$$

In the spherical approximation, $\gamma_{2}=\gamma_{3}$, we find the oscillator strength

$$
f_{0 j}=\frac{2 m_{0}}{\hbar^{2} \gamma_{1}^{0}}\left(E_{j}-E_{0}\right) \frac{1}{2 F_{0}+1} \sum_{F_{0 z}, F_{j z}}\left|\left\langle\Phi_{0}^{I}|z| \Phi_{j}^{I}\right\rangle+\left\langle\Phi_{0}^{E}|z| \Phi_{j}^{E}\right\rangle\right|^{2} .
$$

The summation here is over the degenerate states $F_{0 z}$, of the initial $(0)=\left(n_{0}, L_{0}, F_{0}, F_{0 z}\right)$ level, and states $F_{j z}$ of the final $(j)=\left(n, L, F, F_{z}\right)$ level.

The compuled values of the oscillator strength are very sensitive to the computed envelope functions $[8,9,18,19]$. Within the standard dipole approximation,
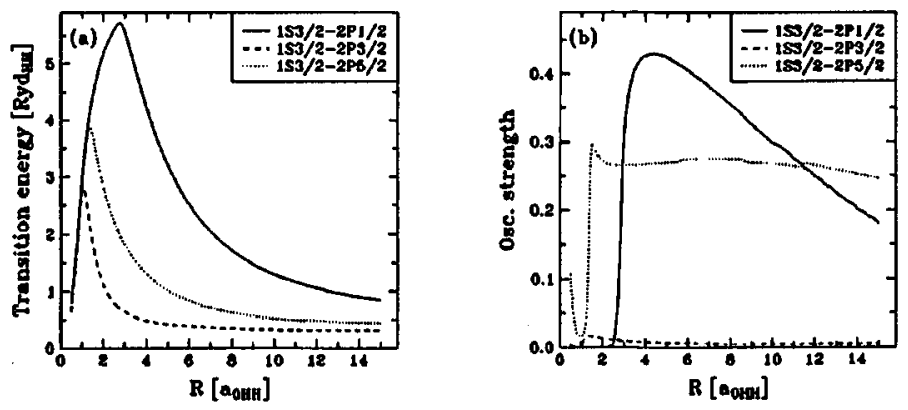

Fig. 2. (a) The photon energies of transitions from the on-center acceptor ground state $1 S$ to the first excited states $2 P$, expressed in units of the rydberg $R y d_{\mathrm{HH}}$ of the heavy hole, as function of the quantum-dot radius, expressed in units of the heavy-hole Bohr radius $a_{0 H н}$. (b) The oscillator strengths for the on-center acceptor $1 S_{3 / 2} \rightarrow 2 P$ transitions as function of the quantum-dot radius, expressed in units of the heavy-hole Bohr radius. The values of the band edge parameters and the height of the potential barrier are the same as in Fig. 1. 
the transitions from the $S$ to $P$ states have large oscillator strengths. We have found particularly large values of the oscillator strengths for transitions from the acceptor ground state to the first excited states. A remarkably large value has been found for the $1 S_{3 / 2} \rightarrow 2 P_{1 / 2}$ transition, as seen in Fig. 2 .

In quantum dots of radii $R$ between $2 a_{0 H I I}$ and $4 a_{0 H I I}$ the oscillator strength for the $1 S_{3 / 2} \rightarrow 2 P_{1 / 2}$ transition is larger than that in the bulk by an order of magnitude, and for the transition $1 S_{3 / 2} \rightarrow 2 P_{5 / 2}$ is larger than that in the bulk by a factor of about 2 . The enhancement is due to a favorable overlap of the envelope functions of the initial and final state involved in the transition. At larger dot radii the oscillator strengths for an acceptor in quantum dot decrease rather slowly with increase in the dot radius. Therefore the enhancement of the oscillator strengths will not be affected by a dispersion of the dot radii values between $R=2 a_{0 H H}$ and $4 a_{0 \mathrm{HH}}$.

\section{Concluding remarks}

We have calculated the energy levels and envelopes of an acceptor situated at the center of a quantum dot. In the spherical approximation the IIamiltonian commutes with the total angular momentum $\boldsymbol{F}$ of the hole. The use of the quantum eigenstates of angular momentum is convenient for calculations of an impurity situated at the center of a spherical quantum dot. In the variational calculation the acceptor envelope functions have been taken as linear combinations of exponential functions allowing analytical expressions for the Hamiltonian matrix elements. By appropriate matching, at the dot heterointerface, the envelope functions and their radial derivatives, the hermiticity of the effective-mass IIamiltonian matrix in cubic semiconductors was ensured, and a set of linear equations was obtained leading to the allowed energy eigenvalues of the lowest states. Examples of the lowest acceptor energy levels and of the oscillator strengths for the dipole transitions have been computed for quantum dots with finite heterointerface potential barriers encountered in typical cubic semiconductors. The oscillator strengths for optical transitions, from the acceptor ground state to the first excited states, computed in dipole approximation, are found to be sensitively dependent on the envelope functions $[18,19]$. The value of the oscillator strength for the optical transition, from the acceptor ground state to one of the excited states, in quantum dot of radius comparable to the acceptor heavy-hole diameter, is enhanced by an order of magnitude with respect to the value for acceptor in the bulk [20]. The electron and the hole states described by angular momentum quantum numbers have been observed in cylindrical quantum dots by photoluminescence measurements $[21,22]$ where the transition energies were increasing at decreasing dot size [21].

This research was supported by grant for the project No. 223469203 of the State Committee for Scientific Research.

\section{References}

[1] A.D. Yoffe, Adv. Phys. 42, 173 (1993).

[2] Jian-Bai Xia, Phys. Rev. B 40, 8500 (1989).

[3] P.C. Sercel, K.J. Vahala, Phys. Rev. B 42, 3690 (1990). 
[4] R. Buczko, Acta Phys. Pol. A 82, 789 (1992).

[5] A. Baldereschi, N.O. Lipari, Phys. Rev. B 8, 2697 (1973).

[6] J.M. Ferreyra, C.R. Proetto, Phys. Rev. B 52, R2309 (1995).

[7] D.A. Varshalovich, A.N. Moskalev, V.K. Khersonskii, Quantum Theory of Angular Momentum, Nauka, Leningrad 1980 (in Russian).

[8] A. Pasquarello, L.C. Andreani, R. Buczko, Phys. Rev. B 40, 5602 (1989).

[9] R. Buczko, F. Bassani, Phys. Rev. B 45, 5838 (1992).

[10] L.R. Ram-Mohan, K.H. Yoo, R.L. Aggarwal, Phys. Rev. B 38, 6151 (1988).

[11] C. Goldoni, A. Fasolino, Phys. Rev. B 51, 9903 (1995).

[12] Landolt-Börnstein, Numerical Dala and Functional Relationships in Science and Technology, New Series, Group III, Vol. 17b (1982) and Vol. 22a (1987), Springer, Berlin.

[13] E.T. Yu, J.O. McCaldin, T.C. McGill, in: Solid State Physics, Vol. 46, Eds. H. Ehrenreich, D. Turnbull, Academic Press, Boston 1992, p. 1.

[14] R.L. Greene, K.K. Bajaj, Phys. Rev. B 31, 6498 (1985).

[15] G. Platero, M. Altarelli, Phys. Rev. B 36, 6591 (1987).

[16] J.W. Wu, A.V. Nurmikko, Phys. Rev. B 38, 1504 (1988).

[17] F. Szmulowicz, G.J. Brown, Phys. Rev. B 51, 13203 (1995).

[18] N. Binggeli, A. Baldereschi, Solid Slate Commun. 66, 323 (1988).

[19] T. Takagahara, Phys. Rev. B 39, 10206 (1989).

[20] A.V. Nurmikko, R.L. Gunshor, Solid State Commun. 92, 113 (1994).

[21] M. Bayer, A. Schmidt, A. Forchel, F. Faller, T.L. Reinecke, P.A. Knipp, A.A. Dremin, V.D. Kulakovskii, Phys. Rev. Lett. 74, 3439 (1995).

[22] L. Samuelson, A. Gustafsson, Phys. Rev. Lett. 74, 2395 (1995). 